\title{
Deuterium at high redshift
}

\section{Primordial abundance in the $z_{\mathrm{abs}}=\mathbf{2 . 6 2 1}$ damped $\mathrm{Ly}-\alpha$ system towards CTQ 247 ${ }^{\star}$}

\author{
P. Noterdaeme ${ }^{1}$, S. López ${ }^{2}$, V. Dumont ${ }^{2}$, C. Ledoux ${ }^{3}$, P. Molaro ${ }^{4}$, and P. Petitjean ${ }^{1}$
}

1 CNRS-UPMC, UMR7095, Institut d'Astrophysique de Paris, 98bis bd Arago, 75014 Paris, France e-mail: noterdaeme@iap.fr

2 Departamento de Astronomía, Universidad de Chile, Casilla 36-D, Santiago, Chile

3 European Southern Observatory, Alonso de Córdova 3107, Vitacura, Casilla 19001, Santiago 19, Chile

${ }^{4}$ Osservatorio Astronomico di Trieste, via G. B. Tiepolo 11, 34131 Trieste, Italy

Received 21 April 2012 / Accepted 17 May 2012

\begin{abstract}
We report the detection of neutral deuterium in the low-metallicity damped Lyman- $\alpha$ system at $z_{\mathrm{abs}}=2.621$ towards the quasar CTQ 247. Using a high signal-to-noise and high spectral resolution $(R=60000)$ spectrum from the Very Large Telescope Ultraviolet and Visual Echelle Spectrograph, we precisely measure the deuterium-to-oxygen ratio $\log N(\mathrm{D} \mathrm{I}) / N(\mathrm{O} \mathrm{I})=0.74 \pm 0.04$, as well as the overall oxygen abundance, $\log N(\mathrm{O} \mathrm{I}) / N(\mathrm{HI})=-5.30 \pm 0.10$ (or equivalently $[\mathrm{O} / \mathrm{H}]=-1.99 \pm 0.10$ with respect to the solar value). Assuming uniform metallicity throughout the system, our measurement translates into $(\mathrm{D} / \mathrm{H})=\left(2.8_{-0.6}^{+0.8}\right) \times 10^{-5}$. This ratio is consistent within errors $(<0.4 \sigma)$ with the primordial ratio, $(\mathrm{D} / \mathrm{H})_{\mathrm{p}}=(2.59 \pm 0.15) \times 10^{-5}$, predicted by standard Big-Bang nucleosynthesis using the WMAP7 value of the cosmological density of baryons $\left(100 \Omega_{\mathrm{b}} h^{2}=2.249 \pm 0.056\right)$. The D I absorption lines are observed to be broader than the $\mathrm{O}_{\mathrm{I}}$ absorption lines. From a consistent fit of the profiles we derive the turbulent broadening to be $5.2 \mathrm{~km} \mathrm{~s}^{-1}$ and the temperature of the gas to be $T=8800 \pm 1500 \mathrm{~K}$, corresponding to a warm neutral medium.
\end{abstract}

Key words. cosmology: observations - primordial nucleosynthesis - quasars: absorption lines - ISM: abundances quasars: individual: J 040718-441014

\section{Introduction}

The primordial abundance of deuterium is fixed by Big-Bang nucleosynthesis (BBN) and directly depends on the baryon-tophoton ratio $\eta$. The $(\mathrm{D} / \mathrm{H})$ ratio hence provides a baryometer of choice (e.g. Steigman 2007). Because of no (or only very marginal) subsequent production of deuterium (e.g. Epstein et al. 1976; Prodanović \& Fields 2003), and because this species is easily destroyed inside stars (astration), (D/H) is expected to decrease monotonically with cosmic time. To probe this scenario, much effort has been devoted to the measurement of the deuterium abundance, in particular in low-metallicity environments at high redshift $(z>2)$. This is done from observations of D I and $\mathrm{H}_{\mathrm{I}}$ absorption lines in QSO absorption systems (Adams 1976). Unfortunately, measurements are not yet possible in the low-density Ly- $\alpha$ forest because of the extremely low D I column densities expected (there is a five order of magnitude difference in the abundance of the two hydrogen isotopes), so one has to turn to higher column density systems, namely Lyman-limit systems $\left(\log N(\mathrm{HI})\left(\mathrm{cm}^{-2}\right)>17\right)$ and damped Lyman- $\alpha$ systems (DLAs, $\log N(\mathrm{HI}) \geq 20.3$ ). Although DLAs are known to be related to star-forming regions, the deuterium abundance is expected to remain close to the primordial value in the low-metallicity regime (Romano et al. 2006). The main problem is then the small velocity separation between $\mathrm{D}_{\mathrm{I}}$ and H I lines, $\Delta v \sim 80 \mathrm{~km} \mathrm{~s}^{-1}$, which causes D I lines to be easily lost within much stronger $\mathrm{H}$ i profiles. It may also be difficult to discern between D I lines and unrelated absorptions from the

\footnotetext{
* Based on archival ESO data Prgm. ID. 70.A-0017(A).
}

Ly- $\alpha$ forest (e.g. Steigman 1994). This explains why few robust measurements have been performed so far (Pettini et al. 2008).

In this Letter, we present a new measurement of the deuterium abundance at high- $z$, in the $z_{\mathrm{abs}}=2.621$ DLA towards CTQ 247 (also called Q 0405-443 or J 040718-441014). This system was first reported by López et al. (2001) from lowresolution spectroscopy observations, together with two other DLAs along the same line of sight (at $z_{\mathrm{abs}}=2.551$ and 2.595). Ledoux et al. (2003) subsequently confirmed the DLAs from high-resolution data but focused mainly on the $z_{\mathrm{abs}}=2.595$ system, in which they detected absorption lines from molecular hydrogen. The metal abundances in the three DLAs were then studied by López \& Ellison (2003). We identified D I absorption lines in the $z_{\mathrm{abs}}=2.621$ system while screening the Very Large Telescope Ultraviolet and Visual Echelle Spectrograph (VLT/UVES) data archive. Interestingly, the system fulfils the optimal criteria for the determination of $(D / H)$ as proposed by York (2002): $N(\mathrm{H} \mathrm{I})$ above $10^{19} \mathrm{~cm}^{-2}$, warm gas (6000-10000 K), wide separation of the D I-bearing component, and availability of $\mathrm{O}_{\mathrm{I}}$ lines.

\section{Observations and data reduction}

CTQ 247 has been observed several times in the past decade using VLT/UVES with different instrument setups and under varying sky conditions. Here, we take advantage of UVES data obtained through programme ID 70.A-0017(A) (P.I. Petitjean) using a $0.8^{\prime \prime}$ slit width and $2 \times 2 \mathrm{CCD}$ pixel binning. This homogeneous dataset is composed of nine exposures totalling more than $12 \mathrm{~h}$ of integration time under good seeing conditions $\left(\sim 0.8^{\prime \prime}\right)$. Dichroic \#1 was used to observe with the blue and red 
spectroscopic arms simultaneously with central wavelengths adjusted to $390 \mathrm{~nm}$ and $570 \mathrm{~nm}$, respectively. Wavelength calibration frames were taken on target using a ThAr lamp immediately after each science exposure. With this setup, a resolving power of $R=60000$ was reached. The spectra were reduced using the UVES data reduction pipeline v4.9.5 based on the ESO Common Pipeline Library v5.3.1. Object and sky were optimally extracted using a properly over-sampled Gaussian or virtual profile depending on the signal-to-noise ratio $(\mathrm{S} / \mathrm{N})^{1}$. This extraction is based on a generalisation of Horne's algorithm to obtain combined optimal sky and object flux estimates via $\chi^{2}$-minimisation. Pixels affected by cosmic ray hits and CCD defects were identified at the same time and removed via $\kappa$-sigma clipping. Individual spectra were shifted to the vacuumheliocentric rest-frame before they were combined, by weighting the flux in each pixel by the inverse of its variance. The resulting spectrum has an $\mathrm{S} / \mathrm{N}$ per pixel of $\sim 55$ at $\lambda_{\text {obs }}=4000 \AA$. Given the exquisite data quality, we were able to identify a slight interorder background over-subtraction at the level of $1 \%$ at $\lambda_{\mathrm{obs}}<$ $3800 \AA$ and increasing towards bluer wavelengths. We corrected for this effet using the bottom of saturated Ly- $\alpha$ forest lines as reference for the true zero level.

\section{Analysis}

We focus on the $z_{\mathrm{abs}}=2.621$ DLA where we detected clear, non-saturated, and unblended DI absorption lines at $z_{\mathrm{abs}}=$ 2.62102, which defines the zero of our adopted velocity scale (see Fig. 1). This corresponds to the bluest component of the O I profile, which spans $\sim 180 \mathrm{~km} \mathrm{~s}^{-1}$. Di lines associated with the other $\left(v>0 \mathrm{~km} \mathrm{~s}^{-1}\right)$ components are in turn lost within the $\mathrm{H}_{\mathrm{I}}$ profile. The total neutral hydrogen column density, $\log N(\mathrm{HI})=20.45 \pm 0.10$, was measured by fitting the damped Ly- $\alpha$ and Ly- $\beta$ lines (Ledoux et al. 2006; López \& Ellison 2003). Since the values from the two studies (also performed using different instruments) agree within 0.02 dex, we did not attempt to repeat this fit and adopted the above $N(\mathrm{HI})$-value.

We used standard multi-component Voigt-profile fitting to derive the column densities of the individual components after normalising the spectrum locally around each line of interest. We simultaneously fitted five O I transitions (at $\lambda=988.5$, 988.6, 988.7, 1039, and 1302) and the Si II $\lambda 1526$ transition over the whole profile, together with Di $\lambda 937$ and DI $\lambda 949$ in the bluest component. The Si II $\lambda 1526$ profile is located outside the Ly- $\alpha$ forest, is not affected by blends, and is characterised by non-saturated components and a high $\mathrm{S} / \mathrm{N}$. We used this transition as a reference to identify the individual components for the absorption lines located in the Ly- $\alpha$ forest and to make a first guess of the redshifts and Doppler parameters. During the fitting process, the Doppler parameters $(b)$ of $\mathrm{O}$ I and $\mathrm{Si}$ II were assumed to be equal. This is the usual assumption in the field and corresponds to $b$ mostly dominated by turbulent motions. However, because deuterium is a very light species, the contribution of thermal broadening, $b_{\text {th }}=\sqrt{2 k T / m}$ (where $k$ is the Boltzmann constant, $m$ the atomic mass, and $T$ the temperature of the gas), is not negligible. Therefore, the Doppler parameter for atomic deuterium has been left free to vary. The best fit is obtained for $b(\mathrm{D} \mathrm{I})=10.1 \mathrm{~km} \mathrm{~s}^{-1}$ while $b=5.8 \mathrm{~km} \mathrm{~s}^{-1}$ for $\mathrm{OI}_{\mathrm{I}}$ and $\mathrm{Si}$ II. This corresponds to a temperature of the gas $T=8800 \pm 1500 \mathrm{~K}$ (see Fig. 2), as expected in warm neutral medium (Petitjean et al. 1992; Wolfire et al. 1995). Temperature

\footnotetext{
${ }^{1}$ See ESO UVES pipeline user manual v19.
}
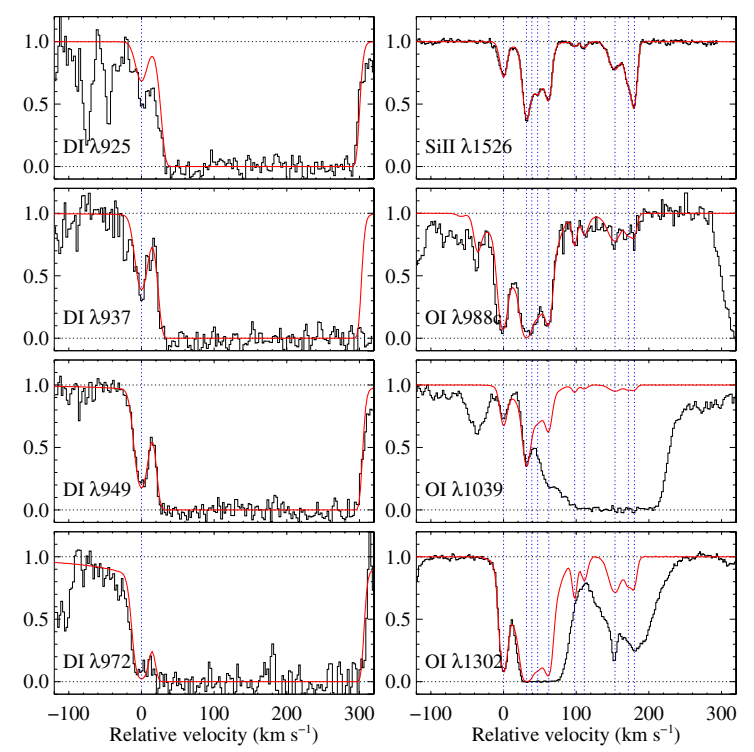

Fig. 1. Velocity plots of $\mathrm{D}_{\mathrm{I}}, \mathrm{O}$ I, and $\mathrm{Si}$ II lines (black). The best-fit synthetic spectrum is superimposed in red. Vertical lines indicate the position of the different components. The profiles for D I $\lambda 925$ and D г $\lambda 972$ were computed using the derived parameters but were not used in the fitting process, the former being contaminated by absorption from the Ly- $\alpha$ forest and the latter being saturated. We also note that the vertical lines and the velocity scale of the Oг $\lambda 988$ panel correspond to the strongest ( $\mathrm{O}_{\mathrm{I}} \lambda$ 988.7) of the three transitions that produce the profile.

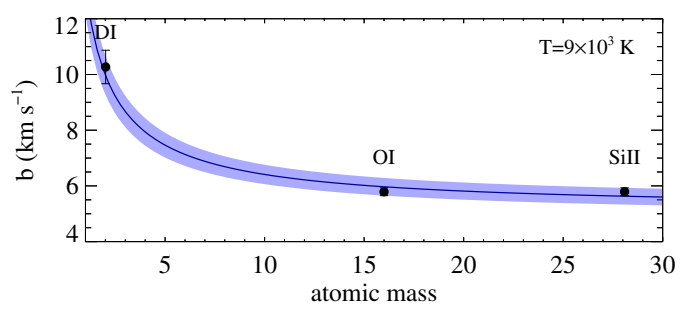

Fig. 2. Doppler parameter as a function of atomic mass. The solid curve and shaded area correspond to the best-fit function $b^{2}=2 \mathrm{kT} / \mathrm{m}+b_{\text {turb }}^{2}$ and $1 \sigma$ range with $b_{\text {turb }}=5.2 \pm 0.2 \mathrm{~km} \mathrm{~s}^{-1}$ and $T=8800 \pm 1500 \mathrm{~K}$.

measurements based on line widths have been difficult in DLAs (see Carswell et al. 2012) because of their components' blending, and are therefore generally limited to narrow lines that sample cold material much less representative of the typical DLAs (Petitjean et al. 2000).

The result of the Voigt-profile fitting is shown in Fig. 1 and the corresponding column densities in Table 1. Formal errors given by fitting procedures $\left(\sigma_{\text {fit }}\right)$ may underestimate the parameters' uncertainties. Therefore, we also performed several fits after shifting the continuum by $\pm 0.5 \sigma$ (where $\sigma$ is the local flux error around each line) and took the difference in extreme values as the error due to uncertainties in the continuum placement, $\sigma_{\text {cont }}$. Errors quoted in the table are estimated as $\sigma_{\text {tot }}^{2}=\sigma_{\text {fit }}^{2}+\sigma_{\text {cont }}^{2}$.

It is not possible to directly measure the $\mathrm{H}_{\mathrm{I}}$ column density in each individual component but we can reasonably assume that $N(\mathrm{HI})$ scales directly with $N(\mathrm{O}$ I) (see Timmes et al. 1997). O I, H I and D I are locked by favourable charge exchange reactions (e.g. Jenkins et al. 2000) so we do not require any photo-ionisation correction. Our assumption therefore simply corresponds to that of a uniform metallicity throughout the profile $^{2}$ (here $[\mathrm{O} / \mathrm{H}]=-1.99$, with respect to the solar value

2 Strictly speaking, we do not even need the metallicity to be equal in all the components, but that the metallicity in the $v=0 \mathrm{~km} \mathrm{~s}^{-1}$ component equals the mean overall value. 
Table 1. Parameters of the modelled absorption profile.

\begin{tabular}{lccccc}
\hline \hline$z$ & $v$ & $b$ & \multicolumn{3}{c}{$\log N\left(\mathrm{~cm}^{-2}\right)$} \\
& $\left(\mathrm{km} \mathrm{s}^{-1}\right)$ & $\left(\mathrm{km} \mathrm{s}^{-1}\right)$ & $\mathrm{O}_{\mathrm{I}}$ & $\mathrm{D}_{\mathrm{I}}$ & $\mathrm{H} \mathrm{I}^{a}$ \\
\hline 2.621022 & 0 & $5.8 \pm 0.1\left(\mathrm{O}_{\mathrm{I}}\right) / 10.3 \pm 0.6(\mathrm{D} \mathrm{I})$ & $14.22 \pm 0.02$ & $14.96 \pm 0.03$ & 19.52 \\
2.621403 & +31 & $6.0 \pm 0.3$ & $14.59 \pm 0.04$ & - & 19.89 \\
2.621492 & +39 & $28.9 \pm 1.1$ & $14.71 \pm 0.03$ & - & 20.01 \\
2.621589 & +47 & $11.0 \pm 2.0$ & $14.05 \pm 0.16$ & - & 19.35 \\
2.621774 & +62 & $5.3 \pm 0.5$ & $14.14 \pm 0.07$ & - & 19.44 \\
2.622204 & +98 & $3.6 \pm 1.4$ & $13.29 \pm 0.09$ & - & 18.59 \\
2.622364 & +111 & $5.3 \pm 3.4$ & $13.14 \pm 0.15$ & - & 18.44 \\
2.622876 & +153 & $11.1 \pm 1.2$ & $13.62 \pm 0.11$ & - & 18.92 \\
2.623095 & +172 & $6.0 \pm 2.1$ & $13.23 \pm 0.20$ & - & 18.53 \\
2.623196 & +180 & $4.1 \pm 0.5$ & $13.12 \pm 0.25$ & - & 18.42 \\
\hline & & & $15.15 \pm 0.02$ & - & $20.45 \pm 0.10$ \\
\hline
\end{tabular}

Notes. ${ }^{(a)}$ Total $N(\mathrm{H}$ I) from Ledoux et al. (2006), distributed over the different components by scaling to $N(\mathrm{O}$ I).

from Lodders 2003). Indeed, the warm phase of DLAs shows a high level of chemical uniformity (Prochaska 2003; Rodríguez et al. 2006), though we caution that this has not yet been tested at metallicities below 1/50th solar. During the fitting process, we therefore modelled the $\mathrm{H}$ i profiles by scaling the column densities with $N(\mathrm{OI})$. The $\mathrm{H}_{\mathrm{I}} b$-values are highly degenerate, in particular in the central components, but they are consistent with those of $\mathrm{O}_{\mathrm{I}}$ with additional thermal broadenings of $\sim 10^{4} \mathrm{~K}$. The modelled H I Ly- $\gamma$, Ly- $\delta$, Ly- $\epsilon$ and Ly- 8 profiles provide a very satisfactory fit to the data, further supporting our above assumption.

\section{Discussion}

Chemical evolution models (e.g. Romano et al. 2006) predict very little astration of deuterium at the metallicity measured here, $[\mathrm{O} / \mathrm{H}] \approx-2$, therefore we can expect the deuterium abundance towards CTQ 247 to be very close to the primordial value. The $\mathrm{D}$ and $\mathrm{O}$ abundances are expected to be anti-correlated, because the former is destroyed in stars while the latter is produced during stellar nucleosynthesis. Here, the $(\mathrm{D} / \mathrm{O})$ ratio is much higher than what is measured locally, but also higher than several measurements at high-z (see Hébrard \& Moos 2003, and references therein). Therefore, this direct measurement alone already indicates very little astration. Furthermore, the lack of odd-even effect (Arnett 1971) in this low-metallicity DLA indicates a low, quiescent star-formation history in the system (López \& Ellison 2003). Indeed, we obtain $(\mathrm{D} / \mathrm{H})=$ $2.8_{-0.6}^{+0.8} \times 10^{-5}$, which agrees remarkably well with the primordial value, $(\mathrm{D} / \mathrm{H})_{\mathrm{p}}=(2.59 \pm 0.15) \times 10^{-5}$, predicted by standard BBN (Coc et al. 2012) using $100 \Omega_{\mathrm{b}} h^{2}=2.249$ from the study of cosmic microwave background (CMB) anisotropies (Komatsu et al. 2011).

In Fig. 3, we compare our abundance measurement to that in other high-redshift quasar absorption systems (Burles \& Tytler 1998a,b; Crighton et al. 2004; Fumagalli et al. 2011; Kirkman et al. 2003; Levshakov et al. 2002; O'Meara et al. 2001, 2006; Pettini \& Bowen 2001; Pettini et al. 2008; Srianand et al. 2010). The scatter between the different measurements is larger than the published errors. This is probably the consequence of underestimated uncertainties. Indeed, the robustness of each $(\mathrm{D} / \mathrm{H})$ measurement has been long discussed in the literature. For example, the value obtained by Crighton et al. (2004) is dependent on the description of the gas kinematics and the error on $N(\mathrm{HI})$ is very likely underestimated (O'Meara et al. 2006).

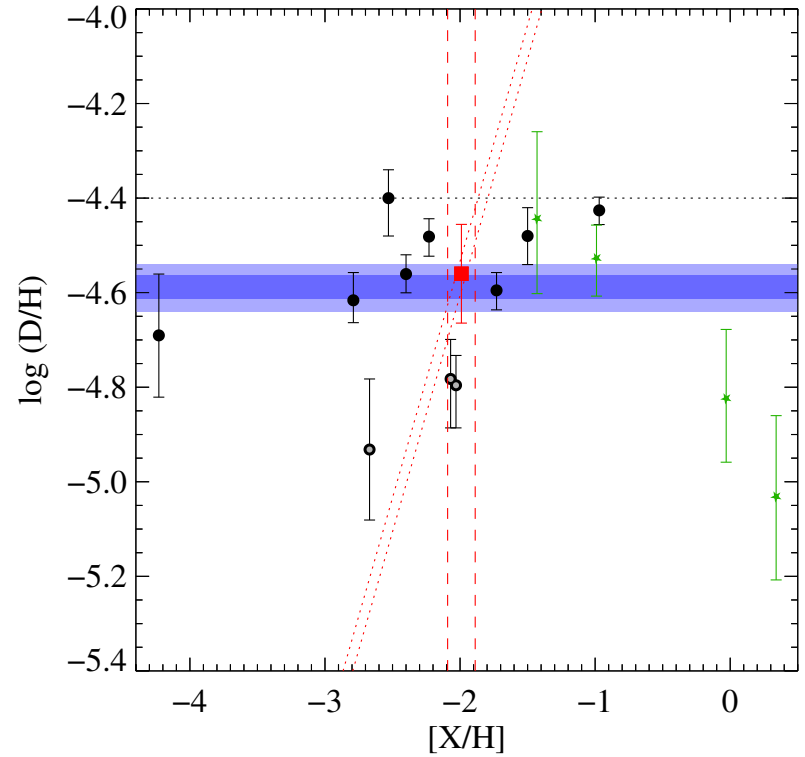

Fig. 3. High-redshift $\mathrm{D} / \mathrm{H}$ values as a function of metallicity $([\mathrm{O} / \mathrm{H}]$ or $[\mathrm{Si} / \mathrm{H}]$ ). Our measurement towards CTQ 247 is shown as a red square. The red dotted lines (resp. vertical dashed lines) represent our constraint on $(\mathrm{D} / \mathrm{O})$ (resp. $[\mathrm{O} / \mathrm{H}])$. Other measurements from the literature are shown as filled circles. Grey filled circles have cautionary remarks in the text (from left to right: Srianand et al. 2010; Crighton et al. 2004; Pettini \& Bowen 2001). Green stars represent $\mathrm{HD} / 2 \mathrm{H}_{2}$ measurements. The blue stripes represent the $1 \sigma$ and $2 \sigma$ prediction on $(\mathrm{D} / \mathrm{H})_{\mathrm{p}}$ from Coc et al. (2012) using standard BBN and $\Omega_{\mathrm{b}} h^{2}$ from WMAP7 (Komatsu et al. 2011). The horizontal dotted line represents the primordial value proposed by Olive et al. (2012), see text.

Pettini et al. (2008) also cautioned that the measurement towards Q 2206-199 (Pettini \& Bowen 2001) may suffer from a poor determination of $N(\mathrm{DI})$ because of the low $\mathrm{S} / \mathrm{N}$ and resolution achieved ${ }^{3}$. Overall, $(\mathrm{D} / \mathrm{H})$ ratios tend to be slightly higher than the standard BBN prediction, with a weighted mean $\langle(\mathrm{D} / \mathrm{H})\rangle=2.94_{-0.34}^{+0.39} \times 10^{-5}$. The same mean was obtained by Pettini et al. (2008), after removing values they considered not robust enough and rescaling the errors to make them consistent with the observed dispersion. These authors subsequently used the $(\mathrm{D} / \mathrm{H})$ ratios to slightly correct downwards $\Omega_{\mathrm{b}}$ from WMAP. Yet, the study of CMB anisotropies now provides very robust

3 This system has been observed using HST-STIS while others were observed using echelle spectrographs on 8-10 m class telescopes. 
estimates of cosmological parameters (Komatsu et al. 2011), in agreement with baryon acoustic oscillation measurements (Percival et al. 2010). It seems therefore more appropriate to seek an alternative explanation for the high $(\mathrm{D} / \mathrm{H})$ values, if these were further confirmed.

Olive et al. (2012) recently proposed cosmological evolution models that alter the nuclear processes during or right after $\mathrm{BBN}$ and which are able to solve the ${ }^{7} \mathrm{Li}$ problem while leading to a higher $(\mathrm{D} / \mathrm{H})_{\mathrm{p}}$, possibly more consistent with the current data (horizontal dotted line in Fig. 3). It is then still possible that the astration of deuterium decreases the $(\mathrm{D} / \mathrm{H})$ ratio locally without necessarily being accompanied by an increased oxygen production (as suggested by Fields et al. 2001; and possibly observed by Srianand et al. 2010 ). Indeed, the astration efficiency depends on the relative stellar populations and the ratio of gas mass to the total galactic mass (e.g. Vangioni-Flam \& Audouze 1988; Vangioni et al. 2011). Local astration could therefore explain the non-primordial $(\mathrm{D} / \mathrm{H})$ ratios, even at low metallicity.

Before discussing the chemical evolution of deuterium abundance in more detail, we note that metallicities are difficult to establish precisely, in particular if elements other than $\mathrm{O}_{\mathrm{I}}$ are used for low $N(\mathrm{H}$ I) systems since ionisation corrections can be uncertain. For example, the extremely low metallicity claimed by Fumagalli et al. (2011) might be underestimated because of the delicate assumption on the ionisation parameter. Some stellar processing of the gas is thus not completely excluded. No decrease of the $(\mathrm{D} / \mathrm{H})$ ratio with metallicity is observed at high redshift for the full range probed by direct $(\mathrm{D} / \mathrm{H})$ measurements up to one tenth solar (see Fig. 3), in agreement with standard chemical evolution models (e.g. Romano et al. 2006). The highmetallicity end, however, could be probed at high-z in molecularrich DLAs using $[\mathrm{S}, \mathrm{Zn} / \mathrm{H}]$ and the $\mathrm{HD} / 2 \mathrm{H}_{2}$ ratio (Noterdaeme et al. 2008, 2010). As expected, the corresponding abundances are significantly lower than the primordial value ${ }^{5}$. Although the difficulties presented by line identification and small numbers of available transitions do not apply in the case of HD-based measurements, poorly known HD-chemistry and self-shielding effects may alter these measurements (Balashev et al. 2010). Nevertheless, measurements performed at lower metallicities (around -1.4, Ivanchik et al. 2010; and -1, Balashev et al. 2010) are very consistent with atomic-based measurements. The HD technique could then be a complementary way to measure the primordial value at $[\mathrm{X} / \mathrm{H}] \leq-1$, while giving constraints on galactic chemical evolution at solar metallicities.

\section{Conclusion}

We reported a new measurement of deuterium abundance in a low-metallicity DLA. The observed $(\mathrm{D} / \mathrm{H})$ value agrees remarkably well with the primordial value predicted by standard Big-Bang nucleosynthesis using the baryon-to-photon ratio derived independently from CMB anisotropies. As noticed by several authors (e.g. Pettini et al. 2008; Ivanchik et al. 2010; Srianand et al. 2010; Olive et al. 2012), the dispersion of other available measurements is higher than the published errors. This could be either observational or physical, where high values could possibly be explained by cosmological evolution models

\footnotetext{
${ }^{4}$ Note, however, that the $N(\mathrm{HI})$ measurement is complex in this system owing to strong blending of the damped lines with other systems.

${ }^{5} \mathrm{HD} / 2 \mathrm{H}_{2}$ values are however above what is expected in closed-box models and could then indicate significant accretion of gas from the intergalactic medium, see discussion in Noterdaeme et al. (2008).
}

including post-BBN processing of the light elements, and where low values would be explained by local astration. Accurately measuring the abundance of deuterium at high redshift is clearly a difficult task and each measurement suffers from its own uncertainties. It is therefore important to obtain new high-redshift measurements - of both $(\mathrm{D} / \mathrm{H})$ and $(\mathrm{D} / \mathrm{O})$ - in systems with different chemical enrichment for a better understanding of deuterium production during $\mathrm{BBN}$ and its astration due to stellar processing in the course of galactic chemical evolution.

Acknowledgements. We thank the referee for helpful comments and suggestions and Elisabeth Vangioni for useful discussions on the BBN. S.L. was supported by FONDECYT grant number 1100214 and V.D. by CONICYT/Gemini Astronomy grant for Master Students at Universidad de Chile number 32100014.

\section{References}

Adams, T. F. 1976, A\&A, 50, 461

Arnett, W. D. 1971, ApJ, 166, 153

Balashev, S. A., Ivanchik, A. V., \& Varshalovich, D. A. 2010, Astron. Lett., 36, 761

Burles, S., \& Tytler, D. 1998a, ApJ, 499, 699

Burles, S., \& Tytler, D. 1998b, ApJ, 507, 732

Carswell, R. F., Becker, G. D., Jorgenson, R. A., Murphy, M. T., \& Wolfe, A. M. 2012, MNRAS, 422, 1700

Coc, A., Goriely, S., Xu, Y., Saimpert, M., \& Vangioni, E. 2012, ApJ, 744, 158

Crighton, N. H. M., Webb, J. K., Ortiz-Gil, A., \& Fernández-Soto, A. 2004, MNRAS, 355, 1042

Epstein, R. I., Lattimer, J. M., \& Schramm, D. N. 1976, Nature, 263, 198

Fields, B. D., Olive, K. A., Silk, J., Cassé, M., \& Vangioni-Flam, E. 2001, ApJ, 563,653

Fumagalli, M., O’Meara, J. M., \& Prochaska, J. X. 2011, Science, 334, 1245

Hébrard, G., \& Moos, H. W. 2003, ApJ, 599, 297

Horne, K. 1986, PASP, 98, 609

Ivanchik, A. V., Petitjean, P., Balashev, S. A., et al. 2010, MNRAS, 404, 1583

Jenkins, E. B., Oegerle, W. R., Gry, C., et al. 2000, ApJ, 538, L81

Kirkman, D., Tytler, D., Suzuki, N., O'Meara, J. M., \& Lubin, D. 2003, ApJS, 149,1

Komatsu, E., Smith, K. M., Dunkley, J., et al. 2011, ApJS, 192, 18

Ledoux, C., Petitjean, P., \& Srianand, R. 2003, MNRAS, 346, 209

Ledoux, C., Petitjean, P., Fynbo, J. P. U., Møller, P., \& Srianand, R. 2006, A\&A, 457,71

Levshakov, S. A., Dessauges-Zavadsky, M., D’Odorico, S., \& Molaro, P. 2002, ApJ, 565, 696

Lodders, K. 2003, ApJ, 591, 1220

López, S., \& Ellison, S. L. 2003, A\&A, 403, 573

López, S., Maza, J., Masegosa, J., \& Marquez, I. 2001, A\&A, 366, 387

Noterdaeme, P., Petitjean, P., Ledoux, C., Srianand, R., \& Ivanchik, A. 2008 , A\&A, 491, 397

Noterdaeme, P., Petitjean, P., Ledoux, C., et al. 2010, A\&A, 523, A80

Olive, K. A., Petitjean, P., Vangioni, E., \& Silk, J. 2012, MNRAS, submitted [arXiv: 1203.5701]

O'Meara, J. M., Tytler, D., Kirkman, D., et al. 2001, ApJ, 552, 718

O’Meara, J. M., Burles, S., Prochaska, J. X., et al. 2006, ApJ, 649, L61

Percival, W. J., Reid, B. A., Eisenstein, D. J., et al. 2010, MNRAS, 401, 2148

Petitjean, P., Bergeron, J., \& Puget, J. L. 1992, A\&A, 265, 375

Petitjean, P., Srianand, R., \& Ledoux, C. 2000, A\&A, 364, L26

Pettini, M., \& Bowen, D. V. 2001, ApJ, 560, 41

Pettini, M., Zych, B. J., Murphy, M. T., Lewis, A., \& Steidel, C. C. 2008, MNRAS, 391, 1499

Prochaska, J. X. 2003, ApJ, 582, 49

Prodanović, T., \& Fields, B. D. 2003, ApJ, 597, 48

Rodríguez, E., Petitjean, P., Aracil, B., Ledoux, C., \& Srianand, R. 2006, A\&A, 446, 791

Romano, D., Tosi, M., Chiappini, C., \& Matteucci, F. 2006, MNRAS, 369, 295

Srianand, R., Gupta, N., Petitjean, P., Noterdaeme, P., \& Ledoux, C. 2010, MNRAS, 405, 1888

Steigman, G. 1994, MNRAS, 269, L53

Steigman, G. 2007, Annu. Rev. Nucl. Part. Sci., 57, 463

Timmes, F. X., Truran, J. W., Lauroesch, J. T., \& York, D. G. 1997, ApJ, 476, 464

Vangioni, E., Silk, J., Olive, K. A., \& Fields, B. D. 2011, MNRAS, 413, 2987

Vangioni-Flam, E., \& Audouze, J. 1988, A\&A, 193, 81

Wolfire, M. G., Hollenbach, D., McKee, C. F., Tielens, A. G. G. M., \& Bakes, E. L. O. 1995, ApJ, 443, 152

York, D. G. 2002, Planet. Space Sci., 50, 1251 